\title{
Effect of Hydrated Ionic Liquid on Photocycle and Dynamics of Photoactive Yellow Protein
}

\author{
Utana Umezaki ${ }^{1}$, Miu Hatakenaka ${ }^{1}$, Kana Onodera ${ }^{2}$, Hiroto Mizutani ${ }^{2}$, Suhyang Kim ${ }^{3}{ }^{\circledR}$, Yusuke Nakasone ${ }^{3}$, \\ Masahide Terazima ${ }^{3}$ and Yoshifumi Kimura ${ }^{1,2, * \mathbb{D}}$
}

1 Department of Molecular Chemistry and Biochemistry, Faculty of Science and Engineering, Doshisha University, Kyotanabe 610-0321, Japan; uu1@rice.edu (U.U.); hatakenaka.miu.63m@st.kyoto-u.ac.jp (M.H.)

2 Department of Applied Chemistry, Graduate School of Science and Engineering, Doshisha University, Kyotanabe 610-0321, Japan; kana.rn6@gmail.com (K.O.); hiroto.m-0822@ezweb.ne.jp (H.M.)

3 Department of Chemistry, Graduate School of Science, Kyoto University, Kyoto 606-8502, Japan; ksuhyang95@hikari.kuchem.kyoto-u.ac.jp (S.K.); nakasone@kuchem.kyoto-u.ac.jp (Y.N.); mterazima@kuchem.kyoto-u.ac.jp (M.T.)

* Correspondence: yokimura@mail.doshisha.ac.jp

check for updates

Citation: Umezaki, U.;

Hatakenaka, M.; Onodera, K.;

Mizutani, H.; Kim, S.; Nakasone, Y.;

Terazima, M.; Kimura, Y. Effect of

Hydrated Ionic Liquid on Photocycle and Dynamics of Photoactive Yellow

Protein. Molecules 2021, 26, 4554.

https://doi.org/10.3390/

molecules26154554

Academic Editor: Jorge F. B. Pereira

Received: 27 May 2021

Accepted: 23 July 2021

Published: 28 July 2021

Publisher's Note: MDPI stays neutral with regard to jurisdictional claims in published maps and institutional affiliations.

Copyright: (c) 2021 by the authors. Licensee MDPI, Basel, Switzerland. This article is an open access article distributed under the terms and conditions of the Creative Commons Attribution (CC BY) license (https:/ / creativecommons.org/licenses/by/ $4.0 /)$.

\begin{abstract}
The mechanism by which proteins are solvated in hydrated ionic liquids remains an open question. Herein, the photoexcitation dynamics of photoactive yellow protein dissolved in hydrated choline dihydrogen phosphate $(\mathrm{Hy}[\mathrm{ch}][\mathrm{dhp}])$ were studied by transient absorption and transient grating spectroscopy. The photocyclic reaction of the protein in Hy[ch][dhp] was similar to that observed in the buffer solution, as confirmed by transient absorption spectroscopy. However, the structural change of the protein during the photocycle in Hy[ch][dhp] was found to be different from that observed in the buffer solution. The known change in the diffusion coefficient of the protein was apparently suppressed in high concentrations of [ch][dhp], plausibly due to stabilization of the secondary structure.
\end{abstract}

Keywords: hydrated ionic liquid; photoactive yellow protein; photocycle; transient grating spectroscopy; conformational change

\section{Introduction}

The interaction between ionic liquids (ILs) and proteins has attracted significant interest. Numerous researchers have studied how proteins are dissolved in ILs and how cations or anions modify the secondary- or higher-order protein structures [1-6]. In particular, the role of the alkyl chain length of the IL cations and their relationship with the Hofmeister series have been discussed using various spectroscopic techniques. Although neat ILs can sometimes stabilize and functionalize proteins, it has been revealed that some classes of ILs enhance the stability of proteins when water is added (hydrated ionic liquids, HyILs). Fujita et al. first reported that a mixture of water with choline dihydrogen phosphate ([ch][dhp]) (see Figure 1) enhanced the storage stability of cytochrome C $[7,8]$. Cytochrome $\mathrm{C}$ could be stored in a mixture of water and [ch][dhp] (Hy[ch][dhp]) for more than 18 months without denaturation. They also analyzed the state of dissolved cytochrome $\mathrm{C}$ in Hy[ch][dhp] by resonance Raman spectroscopy and FT-IR spectroscopy and found that the structure of the protein did not change in Hy[ch][dhp]. The effect of ILs on protein refolding has been discussed in relation to the stability of the proteins in HyILs [9-12]. For example, the concentration of guanidine hydrochloride required to denature myoglobin in solution decreased with the addition of 1-butyl-3-methylimidazolium tetrafluoroborate $\left(\left[\mathrm{Bmim} \mathrm{BF}_{4}\right)\right.$ [10]. On the other hand, ILs with long alkyl chains such as 1-decyl-3-methylimidazolium chloride can induce the refolding of cytochrome $\mathrm{C}$ after denaturation by urea and guanidine hydrochloride [11]. Recently, HyILs have been shown to refold protein aggregation $[13,14]$. Fujita et al. succeeded in refolding recombinant 
protein aggregates from Escherichia coli in Hy[ch][dhp]. Takekiyo et al. reported that heat-aggregated cytochrome $\mathrm{C}$ was refolded in a mixture of water and alkylammonium nitrate [13].
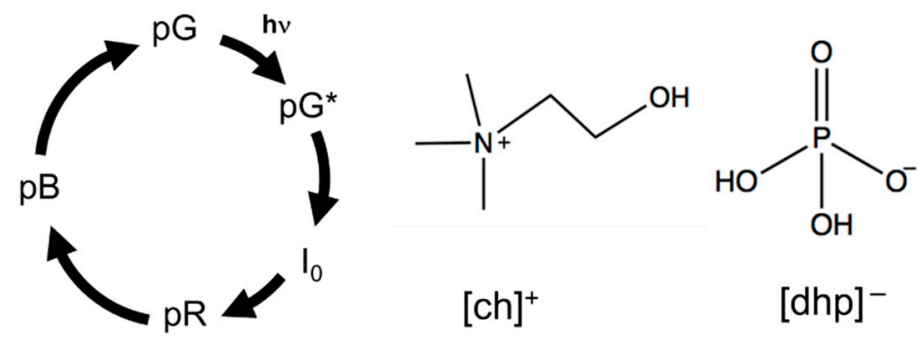

Figure 1. Simplified PYP photocycle model and chemical structure of [ch][dhp].

In the present study, we demonstrate how the photocycle of a protein is modified by Hy[ch][dhp]. There are numerous photoactive proteins, most of which are activated by conformational changes after photoexcitation. In other words, the conformational change of photoactive proteins can be easily controlled by light irradiation, and monitoring their photoreaction in HyILs will reveal the effect of the HyILs on the protein structure. This study aims to clarify how HyILs affect the conformational change of the photoactive protein by monitoring a photoreaction of a photoactive protein in HyILs. To the best of our knowledge, this is the first study on the photocycle of a protein in HyILs. In the experimental design, we have chosen Hy[ch][dhp] as a typical example of HyILs because it has been wildly tested for protein conservation and refolding, as mentioned in the previous paragraph. As a photoactive protein, photoactive yellow protein (PYP) is utilized [15-18]. PYP is a relatively small (14 $\mathrm{kDa})$ water-soluble protein and is considered a blue light photoreceptor for a negative phototactic response. The chromophore of PYP is $p$-coumaric acid (4-hydroxycinnamic acid), which is covalently bonded to the side chain of Cys-69 via a thioester linkage [18-20]. The photocycle of PYP has been studied under various conditions and for various mutants, and various intermediates and detailed kinetics have been proposed. The main photokinetic may be simplified, as shown in Figure 1 [21]. Ground-state PYP (pG state) absorbs light at approximately $440 \mathrm{~nm}$. Upon photoexcitation, $p$-coumaric acid undergoes photoisomerization, which resulted in a shortlived intermediate state $\left(\mathrm{I}_{0}\right)$ within tens of picoseconds with a red-shifted absorption band. This intermediate is converted to a pR state within a nanosecond, which shows the absorption band around $465 \mathrm{~nm}$ [17]. The $\mathrm{pR}$ state is then protonated to produce a $\mathrm{pB}$ state over several hundreds of microseconds. The $\mathrm{pB}$ state is considered as a signaling state, and returns to the original ground state $\mathrm{pG}$ within a few seconds [18].

The structural change of PYP during the photocycle has been studied in detail using various spectroscopic methods such as transient time-resolved infra-red spectroscopy and X-ray crystallography [22-26]. By applying transient grating (TG) spectroscopy together with the transient absorption spectroscopy, Terazima et al. studied the photochemical process from the $\mathrm{pR}$ to $\mathrm{pG}$ state in detail. They found that the diffusion coefficient of the pB state $\left(1.00 \times 10^{-10} \mathrm{~m}^{2} \mathrm{~s}^{-1}\right)$ is smaller than that of the pG state $\left(1.21 \times 10^{-10} \mathrm{~m}^{2} \mathrm{~s}^{-1}\right)$ in buffer solution $[27,28]$. This difference is ascribed to the conformational change of the $\mathrm{N}$-terminal $\alpha$-helices in PYP from $\mathrm{pR}_{1}$ to $\mathrm{pB}[29,30]$. Additionally, they measured the diffusion coefficients of $\mathrm{pG}$ and $\mathrm{pB}$ using N-terminal truncated mutants of PYP [29] and reported that truncation of the $\mathrm{N}$-terminal helices led to little change in the diffusion coefficient of pG vs. pB. Considering FT-IR spectroscopic evidence showing the different interactions between the protein and solvent [31], they concluded that the unfolding of the $\mathrm{N}$-terminal $\alpha$-helix during the photocyclic conversion from $\mathrm{pR}_{2}$ to $\mathrm{pB}$ induced a change in the interaction between the peptide and solvent, which reduced the diffusivity of PYP compared to that in the folded state. It is quite interesting to determine what happens in the photocyclic reaction of PYP in Hy[ch][dhp]. Herein, the photocyclic reaction of PYP in Hy[ch][dhp] with different concentrations of [ch][dhp] is studied. The circular dichroism 
(CD) spectrum of PYP in Hy[ch][dhp] is acquired to investigate the ground-state structure before photoexcitation. Furthermore, transient absorption (TA) and TG spectroscopy are applied to monitor the photoinduced reaction dynamics of PYP in Hy[ch][dhp].

\section{Results}

\subsection{Spectra of $p$ G State in Hy[ch][dhp]}

The absorption profile of the ground state pG did not show any significant change upon addition of [ch][dhp] to the PYP aqueous solution, although the absorption peak shifted to longer wavelength with increasing $w \mathrm{t} \%$ of [ch][dhp] (see Figure S1, Supplementary Materials). This indicates that the chromophore is not removed from the protein even in solutions with a large $w \mathrm{t} \%$ of Hy[ch][dhp]. Similar red-shift of the absorption spectrum has been reported for a mutant of less hydrogen-bonding ability with phenolate of the chromophore [32]. Therefore, [ch][dhp] may relax the hydrogen-bonding structure around the chromophore to some extent. Figure 2 shows the CD spectra of PYP in the pG state in solutions with different $\mathrm{wt}_{\mathrm{t}} \%$ of Hy[ch][dhp] (the weight percentage of [ch][dhp] in solution) in the (a) far-UV region (200-250 nm) and (b) UV-Vis region (250-550 nm). Since the CD intensities in these two regions are different, they were measured at different PYP concentrations ((a) $5.4 \mu \mathrm{M}$ and (b) $103 \mu \mathrm{M}$ ). The spectra obtained in the buffer solution both in (a) and (b) were similar to those reported previously [33,34]. As shown in the figure, the $\mathrm{CD}$ spectrum changed negligibly with increasing [ch][dhp]-to-water ratio. In Figure $2 \mathrm{a}$, the conformational charge of the backbone protein could be estimated. The intensity of the CD peak at $222 \mathrm{~nm}$, which indicates the existence of the $\alpha$-helix, did not show a meaningful change with increasing the concentration of [ch][dhp], although there was a small gap observed in the spectra above the $20 \mathrm{wt} \%$ of [ch][dhp]. The CD spectra from 250 to $550 \mathrm{~nm}$ did not show any change, although the spectrum for the $46 \mathrm{wt} \%$ of [ch][dhp] showed some increase in the intensity at $450 \mathrm{~nm}$. The CD bands from 300 to $500 \mathrm{~nm}$ mostly arise from the chromophore [34], and it can be said that the secondary structure around the chromophore was not affected by [ch][dhp]. From these observations, we can safely conclude that the structure of the pG state of PYP did not show a meaningful change with the addition of [ch][dhp].
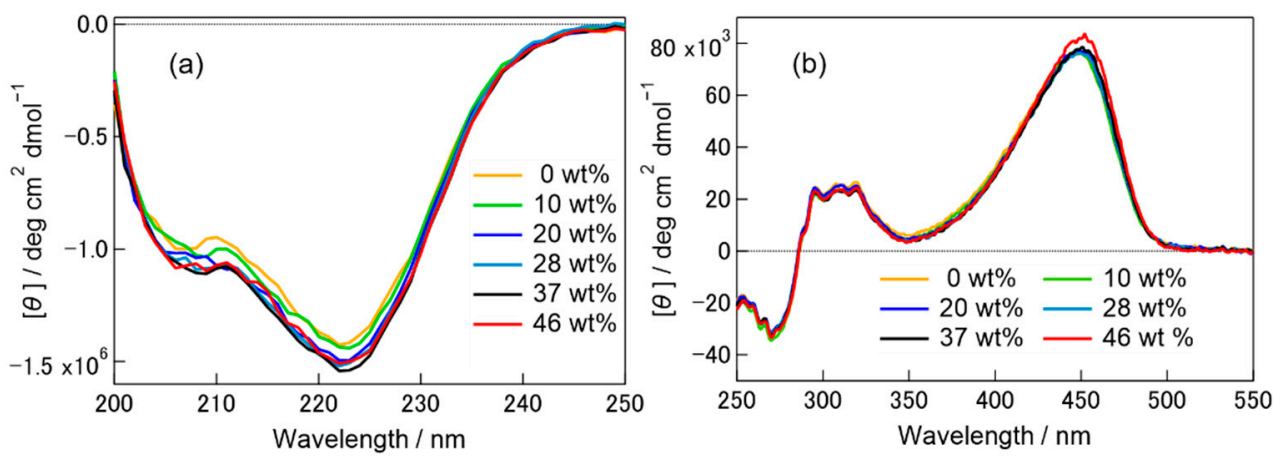

Figure 2. CD spectra of PYP in phosphate buffer ( $\mathrm{pH} 8.0$ ) containing $100 \mathrm{mM} \mathrm{NaCl}$ and various concentrations of $\mathrm{Hy}[\mathrm{ch}][\mathrm{dhp}]$ in (a) 200-250 nm and (b) 250-550 nm. Ellipticity was converted to molar ellipticity per protein.

\subsection{Time Profiles of the Transient Absorption of PYP in Hy[ch][dhp]}

It has been reported that the maximum absorption wavelength of PYP changes during the photocycle in buffer solution: $\mathrm{pG}\left(\lambda_{\max }=446 \mathrm{~nm}\right), \mathrm{pR}\left(\lambda_{\max }=465 \mathrm{~nm}\right)$, and $\mathrm{pB}$ $\left(\lambda_{\max }=355 \mathrm{~nm}\right)$ [17]. By monitoring the transient absorption at $436 \mathrm{~nm}$, which is close to the peak maximum of the $\mathrm{pG}$ state, the depopulation of the $\mathrm{pG}$ state due to conversion to $p B$ via the $p R$ state was analyzed. In the buffer solution, a bleaching signal appeared within a few nanoseconds after illumination with a $460 \mathrm{~nm}$ light. Afterward, a further increase in the bleaching intensity was observed due to the change in the absorption wavelength of the 
$\mathrm{pR}_{2}$ vs. $\mathrm{pB}$ state on the microsecond time-scale. The TA signal $(\Delta \mathrm{OD}(\mathrm{t}))$ can be expressed using a bi-exponential function:

$$
\Delta \mathrm{OD}(t)=\mathrm{a}_{1} \exp \left(-\frac{t}{\tau_{1}}\right)+\mathrm{a}_{2} \exp \left(-\frac{t}{\tau_{2}}\right)
$$

where $\tau_{1}$ is assigned to the time constant of the transformation from $\mathrm{pR}_{2}$ to $\mathrm{pB}^{\prime}$, in which the $\mathrm{N}$-terminal $\alpha$-helix unfolds and $\tau_{2}$ is assigned to the transformation from $\mathrm{pB}^{\prime}$ to $\mathrm{pB}$.

The values of $\tau_{1}$ and $\tau_{2}$ in buffer solution were reported as $170 \mu \mathrm{s}$ and $1.0 \mathrm{~ms}$, respectively $[27,28]$. Simultaneously, upon irradiation with a $460 \mathrm{~nm}$ light, in the short time region where the $\mathrm{pB}^{\prime}$ or the $\mathrm{pB}$ state shows an absorption, an increase in the TA signal around $365 \mathrm{~nm}$ was observed on the same time-scale. Within the time-scale of milliseconds to seconds, the bleaching was reversed due to the recovery of the $\mathrm{pG}$ state (from the $\mathrm{pB}$ state).

Figure 3 shows the short time profiles of the TA signals of PYP, monitored at $436 \mathrm{~nm}$ $(a-c)$ and $365 \mathrm{~nm}(\mathrm{~d}-\mathrm{f})$, with various concentrations of Hy[ch][dhp]. The bleaching signal was observed at $436 \mathrm{~nm}$, and the absorption signal was observed at $365 \mathrm{~nm}$, which indicated that the photocyclic reaction occurred while producing the $\mathrm{pB}$ state, as in the buffer solution. The time profiles of PYP in the $10 \mathrm{wt} \%$ solution were fitted by a bi-exponential function, and the obtained time constants are listed in Table S1 (Figure S2, Supplementary Materials shows the signal in the long time span of the initial beaching signal at $436 \mathrm{~nm}$ ). The TA signals of the protein in the 30 and $49 \mathrm{wt} \%$ solutions were fitted using a single-exponential function. The time constant for the conversion from $\mathrm{pR}$ to $\mathrm{pB}$ was not significantly different from that in the buffer solution. Figure S3, Supplementary Materials shows the recovery of the bleaching signal in the long-time region after irradiation at $436 \mathrm{~nm}$. Recovery of the bleaching signal was also observed in the Hy[ch][dhp] solution, which suggested that the photocyclic reaction was completed in Hy[ch][dhp]. The time constants determined from the fitting are listed in Table S1, where the time constant for recovery to the ground state was slower than that observed in the buffer solution.
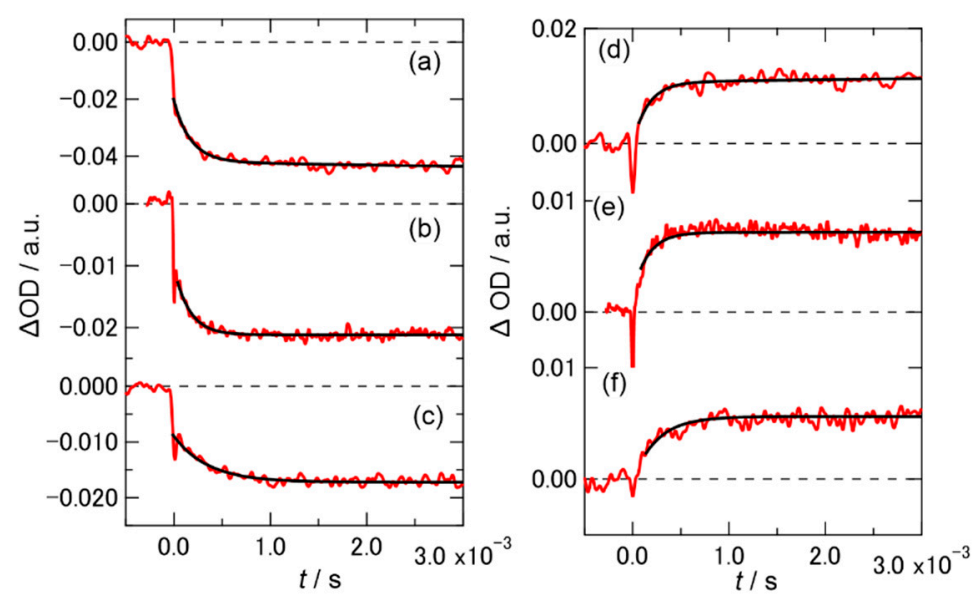

Figure 3. Time profile of the TA at 436 and $365 \mathrm{~nm}$ for PYP in (a,d) $10 \mathrm{wt} \%$, (b,e) $30 \mathrm{wt} \%$, and (c,f) $49 \mathrm{wt} \% \mathrm{Hy}[\mathrm{ch}][\mathrm{dhp}] ;(\mathbf{a}-\mathbf{c}) 436 \mathrm{~nm},(\mathbf{d}-\mathbf{f}) 365 \mathrm{~nm}$. Schemes follow the same formatting.

\subsection{TG Signals of PYP in Hy[ch][dhp]}

Figure 4 shows the TG signals of PYP in different concentrations of Hy[ch][dhp] at similar $q$ values, where $q$ is the grating wavenumber determined by the wavelength of the pump pulsed light and the incident angle between the pulses. The signal obtained in the buffer solution agrees well with that reported previously [28]. Here, the initial peaks of the thermal grating after photoexcitation are not shown in the figure, where only the tails of the thermal grating signal are shown. Compared with the left figure showing the TG signal in the buffer solution, the signals of PYP in Hy[ch][dhp] were significantly different and dependent on the concentration of [ch][dhp], in contrast to the case of the 
TA signals. According to a previous study, the first rise and decay signals were assigned to the population dynamics for conversion of $\mathrm{pR}$ to $\mathrm{pB}$, and the second rise and decay signals were assigned to the diffusion of $\mathrm{pG}$ and $\mathrm{pB}$ [28]. The TG signal $\left(\mathrm{I}_{\mathrm{TG}}(t)\right)$ in the buffer solution was simulated using the following equation:

$$
I_{\mathrm{TG}}(t)=\left[A \exp \left(-D_{\mathrm{th}} q^{2} t\right)+B \exp \left(-k_{B} t\right)+C \exp \left(-k_{C} t\right)+D \exp \left(-D_{\mathrm{pG}} q^{2} t\right)+E \exp \left(-D_{\mathrm{pB}} q^{2} t\right)\right]^{2}
$$

where $D_{\mathrm{th}}$ is the thermal diffusivity; $D_{\mathrm{pB}}$ and $D_{\mathrm{pG}}$ are the diffusion coefficients of the $\mathrm{pB}$ and $\mathrm{pG}$ states, $k_{B}$ and $k_{C}$ represent the rate constants of the conversion from $\mathrm{pR}$ to $\mathrm{pB}$, which were determined from the transient absorption signal $\left(1 / \tau_{1}\right.$ and $1 / \tau_{2}$ in Equation (1)).
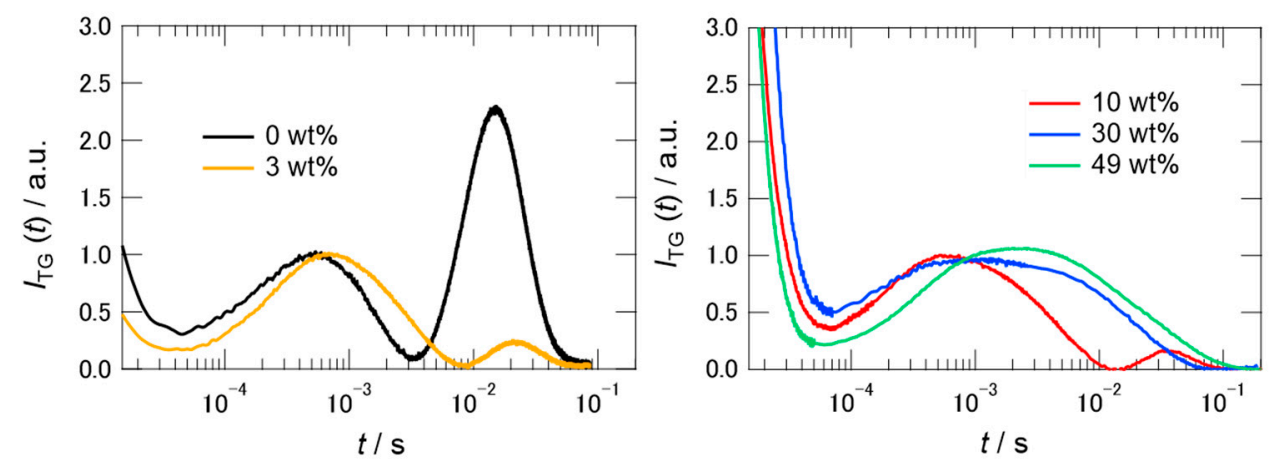

Figure 4. Typical TG signals of PYP in buffer and $3 \mathrm{wt} \%$ of [ch][dhp] (left), and in different wt $\%$ of [ch][dhp] (right). The values of $q^{2}$ are $6.33 \times 10^{11} \mathrm{~m}^{-2}$ (left), and $5.08 \times 10^{11} \mathrm{~m}^{-2}(10 \mathrm{wt} \%)$, $6.60 \times 10^{11} \mathrm{~m}^{-2}(30 \mathrm{wt} \%)$, and $7.20 \times 10^{11} \mathrm{~m}^{-2}(49 \mathrm{wt} \%)$ (right). The signal intensities were normalized relative to the peak at $10^{-3} \mathrm{~s}$.

The signs of the coefficients in moving from $A$ to $E$ were determined by the change in the refractive index for each process, and followed the order: negative, positive, positive, negative, and positive when arranged in time order from fast to slow [28]. Because some components depend on the process of diffusion across the grating (the component including the $q$-dependence), the shape of the signal changed with $q$. As shown in Figure 4, even in $3 \mathrm{wt} \%$ [ch][dhp] solution, the second rise and decay in the figure, representing the diffusion of $\mathrm{pG}$ and $\mathrm{pB}$, became dramatically smaller. The intensity of the signal decreased further in the $10 \mathrm{wt} \%$ solution. In the $30 \mathrm{wt} \%$ solution, the second rise and decay signal vanished, and the shape of the first rise and decay signal changed compared with that in the $10 \mathrm{wt} \%$ solution. A further increase in the concentration of [ch][dhp] did not dramatically change the signal shape, although the signal decayed slightly slower.

To decompose the signal into individual components, several measurements were performed at different $q$-values. Figure 5 summarizes the TG signals of PYP at different $q$ values in different concentrations of [ch][dhp]. As shown in the figure, the shape of the TG signal was dependent on the $q$-value, indicating several contributions to the signal that were not related to the diffusional process. To determine the diffusion coefficients of the $\mathrm{pG}$ and $\mathrm{pB}$ states, global fitting of the TG signals at different $q$ values was performed simultaneously. Equation (2) was used to fit the protein signal obtained in the $10 \mathrm{wt} \%$ solution because the signal shape was the same as that in the buffer solution. In the fitting, we used the time constants of $k_{B}$ and $k_{C}$ as $1 / \tau_{1}\left(5750 \mathrm{~s}^{-1}\right)$ and $1 / \tau_{2}\left(180 \mathrm{~s}^{-1}\right)$ in Equation (1), where these values were determined by TA measurement. However, the third component $C$ in Equation (2) was found to be unnecessary for the fitting, and $C$ was assumed to be zero. The signs of the coefficients from $A$ to $E$ were the same as those in the buffer solution [28]. In the fitting, the relative intensities among pre-exponential coefficients except $A$ were fixed for the signals of different $q$-values. The black curves in the figure show the fitting results, which capture almost all signal traces. From the global fitting, the respective values of $D_{\mathrm{pB}}$ and $D_{\mathrm{pG}}$ in $10 \mathrm{wt} \% \mathrm{Hy}[\mathrm{ch}][\mathrm{dhp}]$ were determined as 
$8.0( \pm 1.2) \times 10^{-11} \mathrm{~m}^{2} \mathrm{~s}^{-1}$ and $9.2( \pm 1.2) \times 10^{-11} \mathrm{~m}^{2} \mathrm{~s}^{-1}$. The parameters obtained from the fit are listed in Table S2, Supplementary Materials.
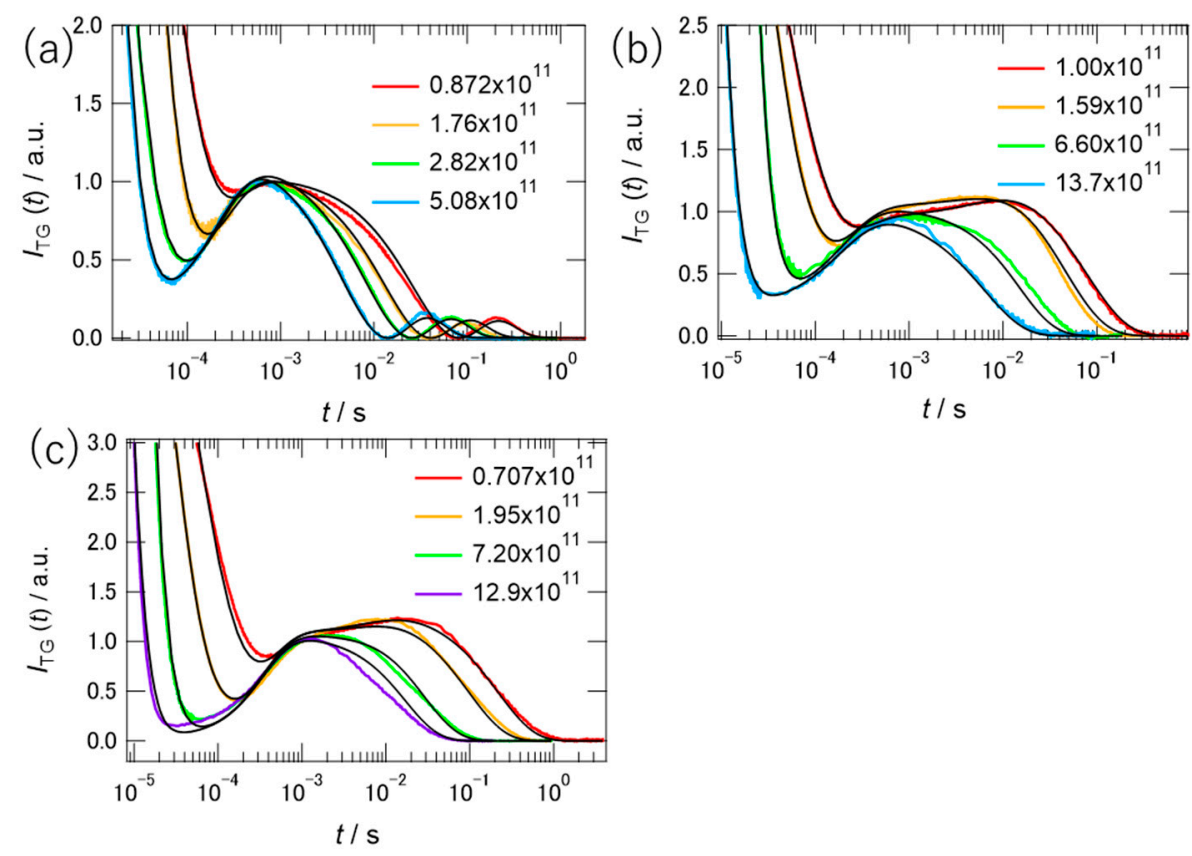

Figure 5. TG signals of PYP in (a) 10, (b) 30, and (c) $49 \mathrm{wt} \%$ of [ch][dhp]. The signal intensities were normalized relative to the peak at $10^{-3} \mathrm{~s}$. The values of $q^{2}$ in $(\mathbf{a}-\mathbf{c})$ are listed in the graph in the unit of $\mathrm{m}^{-2}$. The black curves are fitting curves.

The TG signal shapes were quite different in the 30 and $49 \mathrm{wt} \%$ solutions from those in the buffer and $10 \mathrm{wt} \%$ solutions. At first, we tried to fit the signals to Equation (2), assuming the similar relative amplitudes of pre-exponential coefficients estimated for the signals of $10 \mathrm{wt} \%$ solution. The results of the trail simulations assuming the different diffusion coefficients of the $\mathrm{pB}$ and $\mathrm{pG}$ states like in the buffer solution are shown by the broken curves in Figure S4, Supplementary Materials. Unless we reduced the relative amplitudes of coefficients $D$ and $E$ by a factor of 10, we could not simulate the TG signal at any $q$-value. Inspecting various possibilities of different parameter sets, we found that these signals can be simulated by assuming that the diffusion coefficients of $\mathrm{pB}$ and $\mathrm{pG}$ were very close to each other. Therefore, we assumed that $D_{\mathrm{pB}}$ and $D_{\mathrm{pG}}$ are the same. Under this assumption, the TG signal was modeled by the following equation:

$$
I_{\mathrm{TG}}(t)=\left[A \exp \left(-D_{\mathrm{th}} q^{2} t\right)+B \exp \left(-k_{B} t\right)+C \exp \left(-k_{C} t\right)+D \exp \left(-D_{\mathrm{pGpB}} q^{2} t\right)\right]^{2},
$$

where $D_{\mathrm{pGpB}}$ is the diffusion coefficient of the $\mathrm{pG}$ and $\mathrm{pB}$ states. If we assume the signs of the coefficients $A$ to $D$ as negative, positive, positive, and negative, the simulated signals correspond to the black curves in Figure $5 b, c$.

The simulation works well, although there were some deviations in the intermediate time regions. The rate constant, $k_{B}$, was determined by the transient absorption signal at $6500 \mathrm{~s}^{-1}$ for the $30 \mathrm{wt} \%$ solution and $4000 \mathrm{~s}^{-1}$ for the $49 \mathrm{wt} \%$ solution. Although the slower time constant was not detected using TA, a component of $k_{C}\left(140 \mathrm{~s}^{-1}\right)$ was required to simulate the signal. The relative amplitudes of pre-exponential coefficients are also shown in Table S2. The value of coefficient $D / B$ obtained by the fit to Equation (3) is comparable to the difference between $D / B$ and $E / B$ (Equation (2)) for the $10 \mathrm{wt} \%$ solution, which supports the assumption of the same diffusion coefficients between $D_{\mathrm{pG}}$ and $D_{\mathrm{pB}}$. The obtained diffusion coefficients were $6.4( \pm 0.1) \times 10^{-11} \mathrm{~m}^{2} \mathrm{~s}^{-1}(30 \mathrm{wt} \%)$ and $2.6( \pm 0.1) \times 10^{-11} \mathrm{~m}^{2} \mathrm{~s}^{-1}$ (49 wt\%). 
Before discussing the reaction dynamics of PYP in Hy[ch][dhp], we check two factors which may affect the photocycle of PYP: one is the ionic strength of the solution, and the other is the viscosity of the solvent. The addition of [ch][dhp] to the buffer solution increases the ionic strength of the solution. Borucki et al. reported that the addition of $\mathrm{KCl}$ to a protein solution affects the stability of the intermediate state of the protein [35]. Figure 6a shows the TG signals of PYP in buffer solutions with different concentrations of $\mathrm{NaCl}$. $\mathrm{NaCl}$ solutions with concentrations of $0.55,1.75$, and $3.13 \mathrm{M}$ were prepared, where the concentrations of salt correspond to those of 10, 30, and $50 \mathrm{wt} \% \mathrm{Hy}[\mathrm{ch}][\mathrm{dhp}]$. The intensity of the signal due to mass diffusion decreased with increasing salt concentration. However, the signal persisted, even at high salt concentrations $(3.13 \mathrm{M})$, unlike in the case of the Hy[ch][dhp] solution. The TA signal was also acquired in $1.75 \mathrm{M} \mathrm{NaCl}$ solution, where it was found that a similar photocyclic reaction occurred, although the rate of conversion from $p B$ to $p G$ was rather fast (see Figure S5 and Table S1, Supplementary Materials). The TG signal in the salt solution $(1.75 \mathrm{M})$ was fitted by Equation (4), where the rate of recovery from $\mathrm{pB}$ to $\mathrm{pG}$ was substituted into Equation (2), as follows:

$$
I_{\mathrm{TG}}(t)=\left[A \exp \left(-D_{\mathrm{th}} q^{2} t\right)+B \exp \left(-k_{B} t\right)+C \exp \left(-k_{C} t\right)+D \exp \left(-D_{\mathrm{pG}} q^{2} t\right)+E \exp \left(-\left(D_{\mathrm{pB}} q^{2}+k_{E}\right) t\right)\right]^{2}
$$

where $k_{B}$ and $k_{C}$ were determined to be 6200 and $430 \mathrm{~s}^{-1}$, respectively, from the TA results.

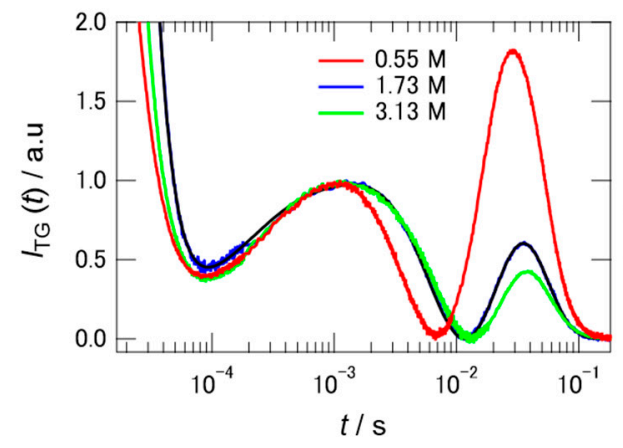

(a)

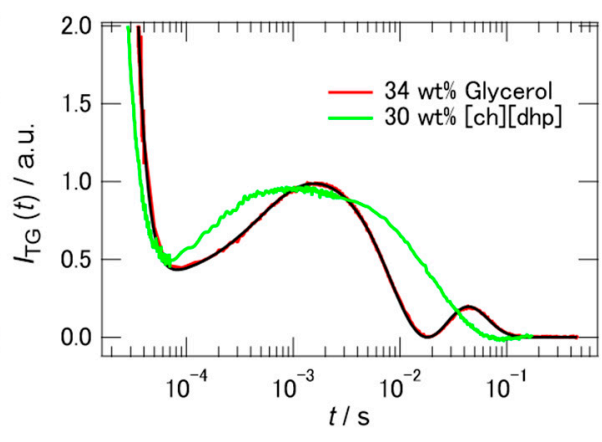

(b)

Figure 6. (a) TG signals of PYP in corresponding molar concentration of $\mathrm{NaCl}$ to 10, 30, $50 \mathrm{wt} \%$ [ch][dhp] solution. The $q^{2}$ values are $5.49 \times 10^{11}(0.55 \mathrm{M}), 4.16 \times 10^{11}(1.73 \mathrm{M})$, and $4.92 \times 10^{11}(3.13 \mathrm{M}) \mathrm{m}^{-2}$. (b) Comparison of the TG signals between in the $34 \mathrm{wt} \%$ glycerol solution $\left(q^{2}=6.90 \times 10^{11} \mathrm{~m}^{-2}\right)$ and in the $30 \mathrm{wt} \%$ [ch][dhp] solution $\left(q^{2}=6.60 \times 10^{11} \mathrm{~m}^{-2}\right)$ at the similar $q$ values. The signal intensities were normalized relative to the peak at $10^{-3} \mathrm{~s}$ both in $(\mathbf{a}, \mathbf{b})$.

The rate of recovery to $\mathrm{pG}\left(k_{E}\right)$ was assumed to be $2.1 \mathrm{~s}^{-1}$ from the amplitudeweighted lifetimes determined from the TA signal. From analysis of the single $q$-trace, the diffusion coefficients of the $\mathrm{pB}$ and $\mathrm{pG}$ states in the salt solution were estimated to be $9.1( \pm 0.5) \times 10^{-11} \mathrm{~m}^{2} \mathrm{~s}^{-1}$ and $11.0( \pm 0.5) \times 10^{-11} \mathrm{~m}^{2} \mathrm{~s}^{-1}$, respectively.

The addition of [ch][dhp] to the buffer solution also increases the viscosity of the solution. Figure S6, Supplementary Materials shows the plots of the viscosity against the [ch][dhp] concentration. As shown in the figure, the viscosity increases with increasing concentration of [ch][dhp], where the viscosity increase is dramatic with more than $25 \mathrm{wt} \%$ of [ch][dhp]. To test the effect of the solvent viscosity, we measured the TG signal in aqueous glycerol solution ( $34 \mathrm{wt} \%$ of glycerol), which shows comparable viscosity ( $2.45 \mathrm{mPa}$ s) with the $30 \mathrm{wt} \% \mathrm{Hy}[\mathrm{ch}][\mathrm{dhp}]$. Figure $6 \mathrm{~b}$ shows the TG signals in both solutions. The TG signals are quite different from each other. The TG signal in aqueous glycerol solution shows a signal similar to the one observed in the buffer solution, although the intensity of the second peak is relatively small. The signal shape clearly suggests that the diffusion coefficient of the $\mathrm{pB}$ state is different from that of the $\mathrm{pG}$ state in the aqueous glycerol solution. The TA signal was also acquired in the aqueous glycerol solution, where it was found that a similar photocyclic reaction occurred, although the rate of conversion from 
pB to pG was fast (see Figure S7 and Table S1, Supplementary Materials). The reduction of the intensity of the second peak is probably due to the fast recovery time from $\mathrm{pB}$ to pG. The TG signal in the aqueous glycerol solution was fitted by Equation (4), as in the case of the salt solution. The values of $k_{B}$ and $k_{C}$ were determined to be 2360 and $240 \mathrm{~s}^{-1}$, respectively, from the TA results. The rate of recovery to $\mathrm{pG}\left(k_{E}\right)$ was assumed to be $6.9 \mathrm{~s}^{-1}$ from the amplitude-weighted lifetimes determined from the TA signal. From analysis of the single $q$-trace, the diffusion coefficients of the $\mathrm{pG}$ and $\mathrm{pB}$ states in the glycerol solution were estimated to be $6.1 \times 10^{-11}$ and $4.4 \times 10^{-11} \mathrm{~m}^{2} \mathrm{~s}^{-1}$, respectively.

\section{Discussion}

The TA results indicate that the photocyclic reaction was completed even in the $49 \mathrm{wt} \%$ $\mathrm{Hy}[\mathrm{ch}][\mathrm{dhp}]$ solution. In other words, the isomerization of $p$-coumaric acid proceeded as in the buffer solutions. However, the TG signals indicate that the protein dynamics, especially the translational diffusion coefficients, are strongly dependent on the concentration of [ch][dhp]. In the buffer solution, the diffusion coefficients of the pB and pG states differ because of the conformational change of the $\mathrm{N}$-terminal $\alpha$-helix. The unfolded $\alpha$-helix undergoes stronger interaction with the solvent molecules, which reduces the diffusion coefficient. On the other hand, in the Hy[ch][dhp] solution at concentrations higher than $30 \mathrm{wt} \%$, we could not simulate the TG signal by using the different diffusion coefficients of the $\mathrm{pB}$ and $\mathrm{pG}$ states. By assuming that $D_{\mathrm{pG}}$ and $D_{\mathrm{pB}}$ are the same, the TG signal could be simulated reasonably. It has been reported that in HyILs, the diffusion coefficient of a protein in its unfolded structure is smaller than that of the protein in its native structure $[9,12]$. Sasmal et al. reported that the diffusion coefficient of human serum albumin is significantly smaller in the unfolded state than in the native form in 1-methyl3-pentylimidazolium bromide with water [9]. Pabbathi and Samanta reported that the diffusion coefficient of an unfolded form of cytochrome $C$ is smaller than that of the native form in hydrated ammonium ILs, although the effect of the ionic concentration was negligible [12]. From these observations, the similar diffusion coefficients of the $\mathrm{pB}$ and $\mathrm{pG}$ states suggests similarity of the conformation of the protein in the two states. According to the CD spectrum shown in Figure 2, the secondary structure of the ground state is not affected by the presence of [ch][dhp]. Therefore, it is reasonable to consider that the $\alpha$-helix is not unfolded in the $\mathrm{pB}$ state and that PYP undergoes a photocyclic reaction with the folded $\alpha$-helix. As mentioned in the introduction, Hy[ch][dhp] stabilizes the protein. In other words, conformational change may be suppressed by [ch][dhp].

The diffusion coefficients of PYP in Hy[ch][dhp] decreased with increasing concentration of [ch][dhp]. The major contribution to this change is the increasing viscosity of the solution with [ch][dhp] (Figure S6). According to the Stokes-Einstein equation, the diffusion coefficient is related to the solvent viscosity $(\eta)$ by the following equation:

$$
D_{i}=\frac{k_{B} T}{6 \pi \eta r}
$$

where $i$ is the chemical species, $k_{B}$ is the Boltzmann constant, $T$ is the absolute temperature, $\eta$ is the viscosity, and $r$ is the hydrodynamic radius.

Although this equation does not necessarily hold due to the complex interaction between the protein and solvent, it may guide the discussion of the viscosity-dependence of the diffusion coefficient.

Figure 7 shows the dependence of the diffusion coefficients on the inverse of the solvent viscosity. The broken line in the figure represents the linear correlation between $D_{\mathrm{pG}}$ and $\eta^{-1}$ using the data for the protein in the 0 and $10 \mathrm{wt} \% \mathrm{Hy}[\mathrm{ch}][\mathrm{dhp}]$ solutions. The diffusion coefficient clearly increases with increasing $\eta^{-1}$, although the dependence is not strictly linear. The diffusion coefficient is faster at high concentrations of Hy[ch][dhp] (smaller $\eta^{-1}$ ) than that predicted from the linear relation (broken line) in low-concentration Hy[ch][dhp] (larger $\eta^{-1}$ ). On the other hand, the diffusion coefficient of the $\mathrm{pB}$ state in the high-concentration $\mathrm{NaCl}$ solution $(1.75 \mathrm{M})$ was smaller than that of the $\mathrm{pG}$ state (Figure 6a). 
This suggests that the unfolded $\alpha$-helix interacts more strongly with the solvent, even if numerous ions are dissolved in the solution. It is also to be noted here that the diffusion coefficient of the $\mathrm{pB}$ state is different from that of the $\mathrm{pG}$ state in the aqueous glycerol solution, which has a similar viscosity to the $30 \mathrm{wt} \%$ [ch][dhp] solution. The diffusion coefficient of the $\mathrm{pG}$ state is somewhat faster than the prediction from the SE prediction, and gives a similar value to that obtained in the $30 \mathrm{wt} \% \mathrm{Hy}[\mathrm{ch}][\mathrm{dhp}]$, while the value of $\mathrm{pB}$ is clearly smaller than the value of pG. Therefore, it can be safely said that the viscosity is not the factor which prohibits the conformational change from $\mathrm{pG}$ to $\mathrm{pB}$.

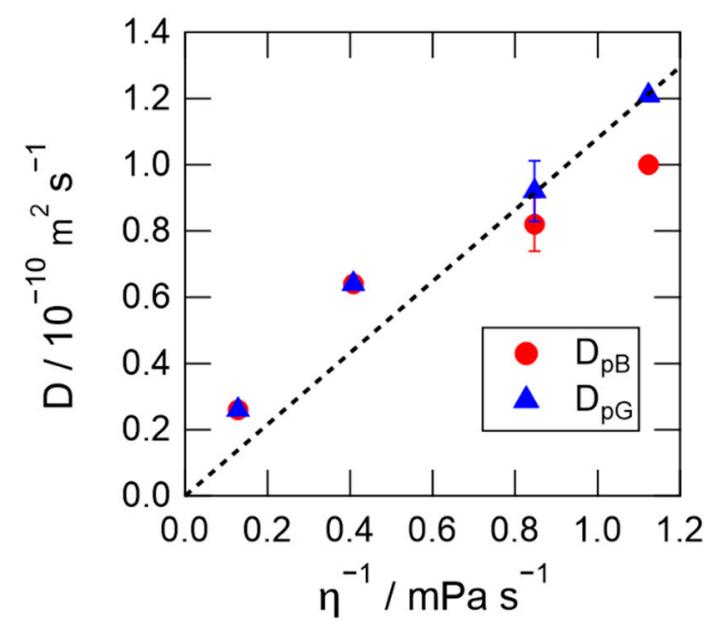

Figure 7. Dependence of diffusion coefficients on the inverse of the viscosity.

Notably, the time for recovery from the $\mathrm{pB}$ to $\mathrm{pG}$ state in $\mathrm{NaCl}$ solution is not very different from that in the buffer solution. The recovery time in the aqueous glycerol solution is faster in buffer solution. On the other hand, the time is much longer in Hy[ch][dhp]. At present, we are not sure of the reason for the difference. It has been reported that the mutation of $\mathrm{Y} 98$ by Q dramatically decelerates the recovery time, where $\mathrm{Y} 98$ is located near the chromophore [35]. Thus, a subtle environmental change around the chromophore as is demonstrated in the red-shift of the absorption spectrum in Hy[ch][dhp] (Figure S1) may affect the time-scale of the photocycle.

\section{Materials and Methods}

Choline dihydrogen phosphate ([ch][dhp]) was synthesized following a previously reported procedure [8]. Briefly, choline chloride (extra pure; Nacalai Tesque Inc., Kyoto, Japan) and potassium hydroxide (>85\%; Sigma-Aldrich Co. LLC, St. Louis, MO, USA) were dissolved in 2-propanol and potassium chloride was precipitated by freezing overnight. The supernatant was removed under reduced pressure. The compound was dissolved in water and converted to [ch][dhp] by adding phosphoric acid ( $>85 \%$; Nacalai Tesque). The water was removed under reduced pressure, and the product was further purified by recrystallization with methanol/acetone. The purity of [ch][dhp] was checked by ${ }^{1} \mathrm{H}-\mathrm{NMR}$ and Mohr's method. H-purity was $>99.5 \%$ and the chloride content was $<0.1 \%$. ${ }^{1} \mathrm{H}-\mathrm{NMR}$ was measured by a JNM-ECA300W (JEOL Ltd., Tokyo, Japan).

PYP was prepared as previously reported [28]. PYP samples in 10, 30, and $49 \mathrm{wt} \%$ Hy[ch][dhp] for the TG measurements were prepared by mixing [ch][dhp] and PYP samples in $10 \mathrm{mM}$ Tris- $\mathrm{HCl}$ with $100 \mathrm{mM} \mathrm{NaCl}$ buffer solution (Tris- $\mathrm{HCl} \mathrm{NaCl} ; \mathrm{pH}=8.0$ ). To acquire the CD spectrum using a circular dichroism spectrometer (J-720WI; JASCO Corp., Tokyo, Japan), PYP solutions of Hy[ch][dhp] in phosphate buffer (pH 8.0) containing $100 \mathrm{mM}$ $\mathrm{NaCl}$ were prepared. For these solutions, the concentration of [ch][dhp] was confirmed from the relative intensity of the ${ }^{1} \mathrm{H}-\mathrm{NMR}$ peak of water vs. that of the cholinium cation. PYP solutions with high salt $(\mathrm{NaCl})$ concentrations comparable to those of Hy[ch][dhp] were prepared similarly. The glycerol solution (34 wt\% of glycerol) of similar viscosity to 
the $30 \mathrm{wt} \% \mathrm{Hy}[\mathrm{ch}][\mathrm{dhp}]$ was prepared by mixing glycerol with the buffer solution. The viscosity of $\mathrm{Hy}[\mathrm{ch}][\mathrm{dhp}], \mathrm{NaCl}$ solutions, and aqueous glycerol solution was determined using a cone-plate-type viscometer (DV-II pro; Brookfield Engineering Laboratories, Inc., Middleboro, MA, USA).

The experimental setup for the transient grating (TG) measurement is described elsewhere [36]. Briefly, an output pulse $(460 \mathrm{~nm})$ of a dye laser (ND6000; Continuum, Milpitas CA, USA) pumped by a third-harmonic pulse $(355 \mathrm{~nm})$ of an Nd:YAG laser (Surelite; Continuum) was used as the excitation pulse. A He-Ne laser (LGK7651-8, 633 nm; Lasos Lasertechnik GmbH, Jena, Germany) was used as the probe beam. The TG signal was detected using a photomultiplier, and the signal was transferred to an oscilloscope (Waverunner 44Xi; Lecroy, Chestnut Ridge, NY, USA). The value of the grating lattice vector was determined by the thermal grating signal decay rate of bromocresol purple in methanol, measured with the same optical geometry, using the values of the thermal diffusivity of methanol. For the transient absorption measurements at 364 and $436 \mathrm{~nm}$, an Hg lamp was used as the light source, and the wavelength was separated by a proper optical band-pass filter. The changes in the optical density with and without the pump pulse $(\Delta \mathrm{OD})$ were measured at each probe wavelength. The TA spectrum $(\Delta \mathrm{OD}(\lambda, t))$ was determined using the following equation:

$$
\Delta \mathrm{OD}(\lambda, t)=-\log \left(\frac{I(\lambda, t)}{I_{0}(\lambda, t)}\right)
$$

where $I_{0}(\lambda, t)$ is the initial probe light intensity without the pump pulse and $I(\lambda, t)$ is the probe light intensity after passing through the sample, which was excited by the pump pulse.

\section{Conclusions}

In the present study, the photocycle of PYP in Hy[ch][dhp] was revealed. Although the photoreaction of the chromophore proceeds as in buffer solution, the protein did not undergo conformational changes in the presence of [ch][dhp]. The present results suggest that [ch][dhp] suppresses the structural changes in proteins, which are closely related to the stability of the proteins in Hy[ch][dhp]. Further studies on the dynamics of the folding or unfolding of proteins are desirable to elucidate the interaction between HyILs and proteins.

Supplementary Materials: The following are available online, Figure S1: UV-Vis absorption spectra of PYP in different wt \% solution of Hy[ch][dhp], Figure S2: time profiles of the bleaching of the transient absorption of PYP in the extended time range, Figure S3: time profiles of the bleaching recovery of the transient absorption of PYP in the long time range, Figure S4: trial fits of the TG signal in the $30 \mathrm{wt} \%$ [ch][dhp] solution using Equation (2), Figure S5: time profile of the transient absorption at $436 \mathrm{~nm}$ for PYP in $1.75 \mathrm{M} \mathrm{NaCl}$ solution, Figure S6: viscosity of Hy[ch][dhp] vs. concentration of [ch][dhp], Figure S7: time profile of the transient absorption at $436 \mathrm{~nm}$ for PYP in $34 \mathrm{wt} \%$ glycerol solution, Table S1: time constants obtained from the transient absorption analysis, Table S2: Parameters obtained and used for the global-fit of the TG signals.

Author Contributions: U.U. and M.H. performed the experiments, analyzed the data and edited the paper. K.O. and H.M. performed the experiments and analyzed the data. S.K., Y.N. and M.T. prepared the protein, supported CD measurement, reviewed and edited the paper, Y.K. conceived and designed the experiments, and wrote the paper. All authors have read and agreed to the published version of the manuscript.

Funding: This work was supported by the MEXT-Supported Program for the Strategic Research Foundation at Private Universities 2015-2019 (S1511025).

Institutional Review Board Statement: Not applicable.

Informed Consent Statement: Not applicable.

Data Availability Statement: The data presented in this study are available on request from the corresponding author. 
Acknowledgments: U.U., M.H. and Y.K. are grateful to N. Higashi and T. Koga (Doshisha University) for the use of the CD spectrometer. Y.K. and M.H. are grateful for the help of experiment to Y. Hatatani (Doshisha University).

Conflicts of Interest: The authors declare no conflict of interest.

Sample Availability: Samples of the compounds are not available from the authors.

\section{References}

1. Fujita, K. Ionic liquids as stabilization and refolding additives and solvents for proteins. Adv. Biochem. Eng. Biotechnol. 2021, 168, 215-226.

2. Kumar, A.; Bhakuni, K.; Venkatesu, P. Strategic planning of proteins in ionic liquids: Future solvents for the enhanced stability of proteins against multiple stresses. Phys. Chem. Chem. Phys. PCCP 2019, 21, 23269-23282. [CrossRef] [PubMed]

3. Patel, A.Y.; Jonnalagadda, K.S.; Paradis, N.; Vaden, T.D.; Wu, C.; Caputo, G.A. Effects of ionic liquids on metalloproteins. Molecules 2021, 26, 514. [CrossRef] [PubMed]

4. Sivapragasam, M.; Moniruzzaman, M.; Goto, M. Recent advances in exploiting ionic liquids for biomolecules: Solubility, stability and applications. Biotechnol. J. 2016, 11, 1000-1013. [CrossRef] [PubMed]

5. Ohno, H.; Fujita, K.; Kohno, Y. Is seven the minimum number of water molecules per ion pair for assured biological activity in ionic liquid-water mixtures? Phys. Chem. Chem. Phys. 2015, 17, 14454-14460. [CrossRef]

6. Weingartner, H.; Cabrele, C.; Herrmann, C. How ionic liquids can help to stabilize native proteins. Phys. Chem. Chem. Phys. 2012, 14, 415-426. [CrossRef]

7. Fujita, K.; MacFarlane, D.R.; Forsyth, M. Protein solubilising and stabilising ionic liquids. Chem. Comm. 2005, 38, 4804-4806. [CrossRef]

8. Fujita, K.; MacFarlane, D.R.; Forsyth, M.; Yoshizawa-Fujita, M.; Murata, K.; Nakamura, N.; Ohno, H. Solubility and stability of cytochrome c in hydrated ionic liquids: Effect of oxo acid residues and kosmotropicity. Biomacromolecules 2007, 8, $2080-2086$. [CrossRef]

9. Sasmal, D.K.; Mondal, T.; Sen Mojumdar, S.; Choudhury, A.; Banerjee, R.; Bhattacharyya, K. An FCS study of unfolding and refolding of CPM-labeled human serum albumin: Role of ionic liquid. J. Phys. Chem. B 2011, 115, 13075-13083. [CrossRef]

10. Fiebig, O.C.; Mancini, E.; Caputo, G.; Vaden, T.D. Quantitative evaluation of myoglobin unfolding in the presence of guanidinium hydrochloride and ionic liquids in solution. J. Phys. Chem. B 2014, 118, 406-412. [CrossRef]

11. Singh, U.K.; Patel, R. Dynamics of ionic liquid-assisted refolding of denatured cytochrome $c$ : A study of preferential interactions toward renaturation. Mol. Pharm. 2018, 15, 2684-2697. [CrossRef] [PubMed]

12. Pabbathi, A.; Samanta, A. On the stability and conformational dynamics of cytochrome $c$ in ammonium ionic liquids. J. Phys. Chem. B 2020, 124, 8132-8140. [CrossRef] [PubMed]

13. Takekiyo, T.; Miyazaki, K.; Watanabe, Y.; Uesugi, Y.; Tanaka, S.; Ishikawa, Y.; Yoshimura, Y. Solubilization and recovery of heat-aggregated cytochrome $c$ using alkylammonium nitrate. J. Mol. Liq. 2019, 291, 111239. [CrossRef]

14. Fujita, K.; Kajiyama, M.; Liu, Y.; Nakamura, N.; Ohno, H. Hydrated ionic liquids as a liquid chaperon for refolding of aggregated recombinant protein expressed in Escherichia coli. Chem. Commun. 2016, 52, 13491-13494. [CrossRef] [PubMed]

15. Meyer, T.E. Isolation and characterization of soluble cytochromes, ferredoxins and other chromophoric proteins from the halophilic phototrophic bacterium Ectothiorhodospira halophila. Biochim. Biophys. Acta Bioenerg. 1985, 806, 175-183. [CrossRef]

16. Meyer, T.E.; Tollin, G.; Hazzard, J.H.; Cusanovich, M.A. Photoactive yellow protein from the purple phototrophic bacterium, Ectothiorhodospira halophila. Quantum yield of photobleaching and effects of temperature, alcohols, glycerol, and sucrose on kinetics of photobleaching and recovery. Biophys. J. 1989, 56, 559-564. [CrossRef]

17. Hoff, W.D.; van Stokkum, I.H.; van Ramesdonk, H.J.; van Brederode, M.E.; Brouwer, A.M.; Fitch, J.C.; Meyer, T.E.; van Grondelle, R.; Hellingwerf, K.J. Measurement and global analysis of the absorbance changes in the photocycle of the photoactive yellow protein from Ectothiorhodospira halophila. Biophys. J. 1994, 67, 1691-1705. [CrossRef]

18. Hellingwerf, K.J.; Hendriks, J.; Gensch, T. Photoactive yellow protein, a new type of photoreceptor protein: Will this "Yellow Lab" bring us where we want to go? J. Phys. Chem. A 2003, 107, 1082-1094. [CrossRef]

19. Baca, M.; Borgstahl, G.E.O.; Boissinot, M.; Burke, P.M.; Williams, D.R.; Slater, K.A.; Getzoff, E.D. Complete chemical structure of photoactive yellow protein: Novel thioester-linked 4-hydroxycinnamyl chromophore and photocycle chemistry. Biochemistry 1994, 33, 14369-14377. [CrossRef]

20. Kort, R.; Vonk, H.; Xu, X.; Hoff, W.D.; Crielaard, W.; Hellingwerf, K.J. Evidence for trans-cis isomerization of the p-coumaric acid chromophore as the photochemical basis of the photocycle of photoactive yellow protein. FEBS Lett. 1996, 382, 73-78. [CrossRef]

21. Mix, L.T.; Carroll, E.C.; Morozov, D.; Pan, J.; Gordon, W.R.; Philip, A.; Fuzell, J.; Kumauchi, M.; van Stokkum, I.; Groenhof, G.; et al. Excitation-wavelength-dependent photocycle initiation dynamics resolve heterogeneity in the photoactive yellow protein from Halorhodospira halophila. Biochemistry 2018, 57, 1733-1747. [CrossRef]

22. Chen, E.; Gensch, T.; Gross, A.B.; Hendriks, J.; Hellingwerf, K.J.; Kliger, D.S. Dynamics of protein and chromophore structural changes in the photocycle of photoactive yellow protein monitored by time-resolved optical rotatory dispersion. Biochemistry 2003, 42, 2062-2071. [CrossRef] [PubMed] 
23. Van Wilderen, L.J.G.W.; van der Horst, M.A.; van Stokkum, I.H.M.; Hellingwerf, K.J.; van Grondelle, R.; Groot, M.L. Ultrafast infrared spectroscopy reveals a key step for successful entry into the photocycle for photoactive yellow protein. Proc. Natl. Acad. Sci. USA 2006, 103, 15050-15055. [CrossRef] [PubMed]

24. Schotte, F.; Cho, H.S.; Kaila, V.R.; Kamikubo, H.; Dashdorj, N.; Henry, E.R.; Graber, T.J.; Henning, R.; Wulff, M.; Hummer, G.; et al. Watching a signaling protein function in real time via 100-ps time-resolved Laue crystallography. Proc. Natl. Acad. Sci. USA 2012, 109, 19256-19261. [CrossRef]

25. Jung, Y.O.; Lee, J.H.; Kim, J.; Schmidt, M.; Moffat, K.; Šrajer, V.; Ihee, H. Volume-conserving trans-cis isomerization pathways in photoactive yellow protein visualized by picosecond X-ray crystallography. Nat. Chem. 2013, 5, 212-220. [CrossRef]

26. Yeremenko, S.; van Stokkum, I.H.; Moffat, K.; Hellingwerf, K.J. Influence of the crystalline state on photoinduced dynamics of photoactive yellow protein studied by ultraviolet-visible transient absorption spectroscopy. Biophys. J. 2006, 90, 4224-4235. [CrossRef]

27. Takeshita, K.; Imamoto, Y.; Kataoka, M.; Mihara, K.I.; Tokunaga, F.; Terazima, M. Structural change of site-directed mutants of PYP: New dynamics during pR state. Biophys. J. 2002, 83, 1567-1577. [CrossRef]

28. Takeshita, K.; Imamoto, Y.; Kataoka, M.; Tokunaga, F.; Terazima, M. Themodynamic and transport properties of intermediate states of the photocyclic reaction of photoactive yellow protein. Biochemistry 2002, 41, 3037-3048. [CrossRef]

29. Khan, J.S.; Imamoto, Y.; Harigai, M.; Kataoka, M.; Terazima, M. Conformational changes of PYP monitored by diffusion coefficient: Effect of N-terminal alpha-helices. Biophys. J. 2006, 90, 3686-3693. [CrossRef]

30. Hoshihara, Y.; Imamoto, Y.; Kataoka, M.; Tokunaga, F.; Terazima, M. Conformational changes in the N-terminal region of photoactive yellow protein: A time-resolved diffusion study. Biophys. J. 2008, 94, 2187-2193. [CrossRef] [PubMed]

31. Xie, A.; Kelemen, L.; Hendriks, J.; White, B.J.; Hellingwerf, K.J.; Hoff, W.D. Formation of a new buried charge drives a largeamplitude protein quake in photoreceptor activation. Biochemistry 2001, 40, 1510-1517. [CrossRef] [PubMed]

32. Changenet-Barret, P.; Plaza, P.; Martin, M.M.; Chosrowjan, H.; Taniguchi, S.; Mataga, N.; Imamoto, Y.; Kataoka, M. Structural effects on the ultrafast photoisomerization of photoactive yellow protein. Transient absorption spectroscopy of two point mutants. J. Phys. Chem. C 2009, 113, 11605-11613. [CrossRef]

33. Harigai, M.; Imamoto, Y.; Kamikubo, H.; Yamazaki, Y.; Kataoka, M. Role of an N-terminal loop in the secondary structural change of photoactive yellow protein. Biochemistry 2003, 42, 13893-13900. [CrossRef]

34. Borucki, B.; Otto, H.; Meyer, T.E.; Cusanovich, M.A.; Heyn, M.P. Sensitive circular dichroism marker for the chromophore environment of photoactive yellow protein: Assignment of the 307 and $318 \mathrm{~nm}$ Bands to the $\mathrm{n} \rightarrow \pi^{*}$ transition of the carbonyl. J. Phys. Chem. B 2005, 109, 629-633. [CrossRef] [PubMed]

35. Borucki, B.; Kyndt, J.A.; Joshi, C.P.; Otto, H.; Meyer, T.E.; Cusanovich, M.A.; Heyn, M.P. Effect of salt and pH on the activation of photoactive yellow protein and gateway mutants Y98Q and Y98F. Biochemistry 2005, 44, 13650-13663. [CrossRef] [PubMed]

36. Nishiyama, Y.; Terazima, M.; Kimura, Y. Charge effect on the diffusion coefficient and the bimolecular reaction rate of diiodide anion radical in room temperature ionic liquids. J. Phys. Chem. B 2009, 113, 5188-5193. [CrossRef] 